\title{
Polar Map
}

National Cancer Institute

\section{Source}

National Cancer Institute. Polar Map. NCI Thesaurus. Code C94956.

A 2-dimensional display in which the value plotted, such as by using a color scale, is located by its radial coordinate and an angular or azimuthal coordinate. 